\title{
INTESTINAL ABSORPTION OF THIAMINE PROPYL DISULFIDE ${ }^{1}$
}

\author{
Biochemical Institute, Kyoto Prefectural University of Medicine, \\ Kawara-machi, Nishijin, Kyoto
}

YOSHITSUGU NOSE AND AKIO IWASHIMA

(Received March 22, 1965)

It is a well-known fact that TPD is superior in intestinal absorption to thiamine hydrochloride. However, some of its mechanism of absorption is still unknown. Recently, Takenouchi et al. (1) infused TPD-S ${ }^{35}$ (outer) into a dog's ligated intestine and assumed, due to the distribution of $\mathrm{S}^{35}$ and thiamine in the mesenteric venous blood, that concurrently with absorption, most of TPD divided into thiamine and $\mathrm{S}^{35}$-propyl radical. On the other hand, Itokawa (2) suggested, based on the fact that thiamine- $\mathrm{S}^{35}$ was observed considerably in the blood of the rabbit after oral administration of TPD-S ${ }^{35}$ (inner), that TPD was absorbed unchanged from the intestine.

In order to decide whether TPD is absorbed from the intestine without being divided into thiamine and propyl radical (without reduction), it is necessary to detect and determine TPD in the portal blood. It was found difficult because TPD was very rapidly reduced, liberating thiamine in the blood. Teraoka (3) and Matsubara (4) suggested, due to the chemical quantitative method using the survived or ligated intestine of the dog, that TPD remained in the perfusion blood or the mesenteric venous blood. In the present paper, the authors studied intestinal absorption of TPD using Yoshimura's method (5) in which the mesenteric venous blood of the dog's ligated intestine was rapidly collected.

\section{EXPERIMENTAL}

\section{Intestinal Ligation and Collection of the Mesenteric Venous Blood}

The dog, weighing about $10 \mathrm{~kg}$, was anesthetized with urethane intravenously. Concurrently, heparin was injected and laparotomy was done. The intestine was ligated in two portions at about $30 \mathrm{~cm}$ intervals at the inferior ileum. The venous blood circulating this portion was dropped and collected through a canula inserted into the periphery of the mesenteric vein. TPD (equivalent to $40 \mathrm{mg}$ thiamine) dissolved in $20 \mathrm{ml}$ saline solution was infused into this ligated portion, and the blood was collected at fixed intervals.

As shown in the experimental data, TPD in the blood was rapidly reduced even when at low temperature. As it was stable in metaphosphoric acid, the blood

1 Following abbreviations is used: TPD, thiamine propyl disulfide.

2 能勢善嗣, 岩島昭夫. 
was so arranged as to drop in cold metaphosphoric acid (2\% in final concentration) or alcohol. In case of separating the blood cell and serum, the blood was directly collected in a centrifuge tube with conical tip. All procedures were carried out in a cold room at below $5^{\circ}$.

\section{Determination of Thiamine and TPD}

Free thiamine was determined by a conventional thiochrome method. TPD was determined according to the allithiamine method (6) by reduction of the metaphosphoric acid-treated solution with cysteine. For assay of TPD, the metaphosphoric acid-extract was treated with cysteine, whereby TPD was completely reduced to thiamine. The difference of the thiamine values with and without cysteine treatment corresponds to TPD (6). For thiamine determination of the blood cells and intestinal wall the vitamin was extracted by heat.

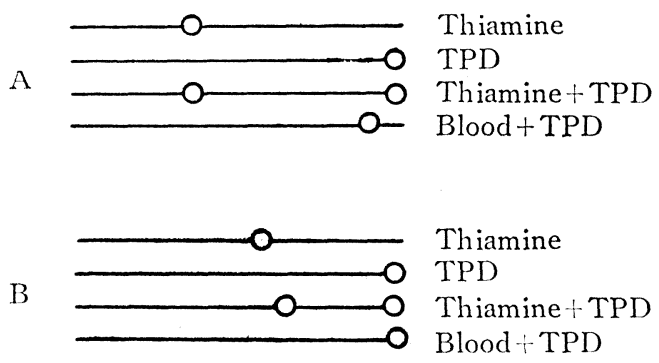

FIG. 1 Detection of TPD in the Blood by Bioautography Using Thiamine-Less Mutant of E. coli

To $2 \mathrm{ml}$ of blood were added $1 \mathrm{ml}$ of $100 \mu \mathrm{g} / \mathrm{ml} \mathrm{TPD}, 5 \mathrm{ml}$ of water and $2 \mathrm{ml}$ of $10 \%$ metaphosphoric acid and the supernatant after centrifugation (pH 5) was spotted.

A, development with butanol-acetic acid-water $(4: 1: 5)$; $\mathrm{B}$, development with $n$-propanol-0.5M acetate buffer, $\mathrm{pH} 4.5(3: 2)$.

\section{Detection of TPD by Bioautography}

A metaphosphoric acid-treated solution was concentrated by lyophilization, and an ethanol-treated solution was spotted directly on Toyo filter paper No. 51 and was developed by $n$-butanol-acetic acid-water $(4: 1: 5)$ or $n$-propanol-0.5 $M$ acetate buffer solution ( $\mathrm{pH}$ 4.5) (3:2) using the ascending method. After drying, TPD was detected by bioautography using a thiamine-less mutant (70-23) of Escherichia coli (7). After development, if cysteine solution was sprayed on a filter paper in advance, TPD was highly sensitive to the bacillus. It was confirmed in the preliminary experiment that TPD added to blood was detected well if the above-mentioned method was used after metaphosphoric acid treatment.

\section{RESULTS}

1. Reduction of TPD by Blood and Metaphosphoric Acid-Treated Solution TPD annexed with blood caused a rapid reduction when it was kept warm. 
The experiment was conducted at low temperature to prevent the reduction of TPD. Considerable reduction was observed even at low temperature as shown in Fig. 2. In a metaphosphoric acid-treated solution ( $\mathrm{pH}$ adjustcd to 6.5), it was comparatively stable and a $14.8 \%$ reduction was seen after 30 minutes. Accordingly, TPD after absorption should be rapidly treated with metaphophoric acid, and a prompt procedure of thiamine determination is required.

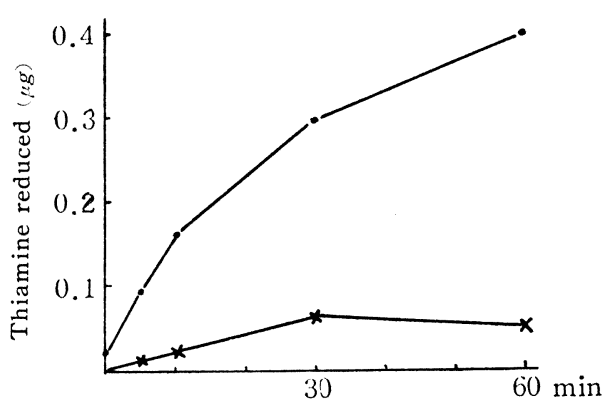

FIG. 2 Reduction of TPD by Whole Blood and the Supernatant after Deproteinization with Metaphosphoric Acid

-, whole blood; $\times$, deproteinized solution.

\section{TPD in the Mesenteric Venous Blood}

Immediately, 1, 5 and 15 minutes after TPD was infused, $5 \mathrm{ml}$ blood was collected from a canula, and the amounts of TPD and thiamine were measured.

In large doses of TPD (equivalent to $40 \mathrm{mg}$ thiamine) most of thiamine in blood was considered to be in the free form, so that determination of the ester form was omitted. As shown in Table 1, in two examples, absorption of the vitamin reached to its maximum 5 minutes after infusion, as has been reported so far. In the method of cysteine reduction, however, the value of TPD was maximum 1 minute after infusion, being $25 \%$ of the absorbed thiamine. The level of TPD decreased gradually 5 and 15 minutes later. Absorption of thiamine was observed soon after

TABLE 1

Reduction of TPD by Whole Blood and Deproteinized Extract

\begin{tabular}{|c|c|c|c|c|c|}
\hline & \multicolumn{5}{|c|}{ Reduction of TPD after min } \\
\hline & 0 & 5 & 10 & 30 & 60 \\
\hline & \multicolumn{5}{|c|}{ per cent } \\
\hline Whole blood $a$ & 7.4 & 21.7 & 36.3 & 67.9 & 91.0 \\
\hline Deproteinized extract ${ }^{b}$ & 0 & 1.9 & 3.7 & 14.8 & 11.0 \\
\hline
\end{tabular}

a To $6 \mathrm{ml}$ of blood was added $4 \mu \mathrm{g}$ TPD. The whole was left standing for various periods. Then the extract after deproteinization was treated with cysteine and the amount of thiamine was determined. From these values the rate of reduction was calculated.

${ }^{b}$ To the deproteinized solution of blood $(80 \mathrm{ml}, \mathrm{pH} 6.5), 5 \mu \mathrm{g}$ of TPD was added and the whole was left standing in an ice water bath and the thiamine produced by reduction was determined. 
TPD was infused, but TPD was not detected in the chemical assay.

Thiamine content of the intestinal wall was also measured. After blood was collected, the small intestine was removed and washed in saline solution. $1 \mathrm{~g}$ of the piece was homognized and extracted for 15 minutes at $80^{\circ}$. Accordingly, no TPD was detected, but the thiamine value was maximum 5 minutes after infusion.

\section{Thiamine Distribution in Blood Cells and Serum}

Blood, $10 \mathrm{ml}$, was collected 5 minutes after infusion, centrifuged and the cells and serum were separated. The cells were washed 3 times with saline solution. Washing water was added to serum,- and both TPD and thiamine were determined. For determining the thiamine in blood cells water was added to make an initial volume and extracted for 15 minutes at $80^{\circ}$ and determined. As shown in Table 2, considerable amounts of thiamine were detected in the blood cells. In the process of separating the blood cells and serum, no TPD was detected in either of them.

TABLE 2

Time Course of the Levels of Free Thiamine and TPD in the Mesenteric Venous Blood after Injection of TPD

A. Amount of thiamine in $5 \mathrm{ml}$ blood

\begin{tabular}{lccccc}
\hline & \multirow{2}{*}{ Before } & Immediately & \multicolumn{3}{c}{ After } \\
\cline { 5 - 6 } & & & $1 \mathrm{~min}$ & $5 \mathrm{~min}$ & $15 \mathrm{~min}$ \\
\hline $\begin{array}{l}\text { Free thiamine }(\mu g) \\
\begin{array}{l}\text { Free thiamine } \\
+ \text { TPD }(\mu g)\end{array}\end{array}$ & 0.10 & 1.6 & 18.0 & 64.0 & 62.4 \\
TPD $(\mu g)$ & - & 1.6 & 24.0 & 68.0 & 64.0 \\
$\begin{array}{l}\text { Percentage of TPD } \\
\text { Thiamine in } \\
\text { intestinal wall }(\mu g / g)^{a}\end{array}$ & & & 6.0 & 4.0 & 1.6 \\
$\begin{array}{l}\text { Free thiamine }+ \text { TPD } \\
\text { in intestinall wall }(\mu g / g)\end{array}$ & & & 25 & 5.8 & 2.5 \\
\hline
\end{tabular}

a After homogenization, thiamine was extracted at $85^{\circ}$ for $15 \mathrm{~min}$.

B. Amount of thiamine in cells and plasma in $10 \mathrm{ml}$ blood

\begin{tabular}{lccccc}
\hline \hline & \multicolumn{2}{c}{ Before } & \multicolumn{2}{c}{ After 5 min } \\
& Cells & Plasma & Cells & Plasma \\
& & & $\mu g$ & & \\
$\begin{array}{l}\text { Free thiamine } \\
\text { Free thiamine+ TPD }\end{array}$ & 0.03 & 0.2 & 8.8 & 132 \\
Total thiamine & - & - & -6.6 & - \\
\hline
\end{tabular}

\section{Bioautography of TPD in the Blood Sample}

The blood collected at fixed intervals, after infusion of TPD, was treated with metaphosphoric acid or ethanol, and was subjected to bioautography. The result illustrated in Fig. 3 was obtained by development with $n$-propanol- $0.5 M$ acetic acid buffer solution. In each case, TPD was detected well. This bacillus was so sensitive to thiamine and TPD that the latter was detected even in the blood immediately after infusion which could not be observed in the chemical determination. 

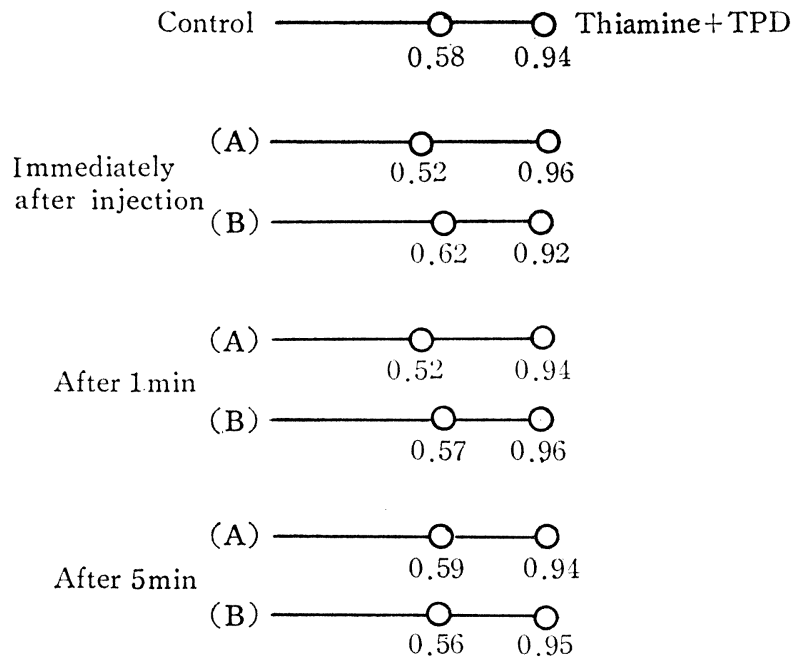

FIG. 3 Detection of TPD in Blood

Immediately, 1 and $5 \mathrm{~min}$ after injection of TPD, the blood was collected. $10 \mathrm{ml}$ of blood was added to $40 \mathrm{ml}$ of ice-cold $2.5 \%$ metaphosphoric acid or $99.5 \%$ ethanol. The deproteinized extract was subjected to paper chromatography.

A, treated with metaphosphoric acid;

$\mathrm{B}$, treated with ethanol.

\section{DISCUSSION}

Intestinal absorption of TPD was investigated in the ligated intestine of the dog. TPD was well detected if the treatment before and after the collection of blood was carried out promptly at low temperature with a rapid quantitative procedure. It was suggested that large amounts of TPD were absorbed from the intestine without reduction. Theoretically, larger amounts of TPD seemed to be absorbed than that obtained actually from the present experiment. It was also presumed that some of TPD would liberate thiamine when it stopped or passed through the intestinal wall, because large amounts of TPD were detected in the blood after the short period of infusion and it decreased gradually with time.

The reason why TPD is superior in intestinal absorption to thiamine hydrochloride seems to result from its high concentration in the intestinal wall and easiness of absorption without being reduced.

\section{SUMMARY}

1. Thiamine propyl disulfide (TPD) is rapidly reduced in blood even at low temperature while it is relatively stable in a metaphosphoric acid-treated solution of blood. 
2. The content of TPD in the mesenteric venous blood was maximum 1 minute after infusion into a ligated intestine, showing $25 \%$ absorption by a method of TPD determination. The value decreased with time.

3. The existance of TPD in the mesenteric venous blood was demonstrated bioautographically using a thiamine-less mutant $(70-23)$ of E. coli.

\section{ACKNOWLEDGEMENT}

The author are indebted to Dr. Hisato Yoshimura and Dr. Kunio Yoshikawa, Department of Physiology, Kyoto Prefectural University of Medicine, for their kind help for the intestine ligation method.

\section{REFFRENCES}

1. Takenouchi, K., Aso, K. and Kobayashi, T., Vitamins 26, 245 (1962).

2. Itokawa, Y., Vitamins 28, 568 (1963).

3. Teraoka, H., Seikagaku 25, 408 (1963).

4. Matsubara, K., Vitamins 12, 80 (1957).

5. Yoshimura, H, Vitamins 27, 312 (1963).

6. Tamaki, T. and Nose, Y., J. Vitaminol. 1, 85 (1955).

7. Okada, Y. and Mori, K., Vitamins 21, 281 (1960). 